\title{
A 36-Year-Old Woman with a History of Dextrocardia and Dyspnea
}

\author{
Kiran K. Devulapally Nitin Bhatt James N. Allen \\ Division of Pulmonary, Allergy, Critical Care and Sleep Medicine, Columbus, Ohio, USA
}

\section{Case Report}

A 36-year-old woman with a history of dextrocardia presented to the clinic for evaluation of several months of exertional dyspnea. She denied any cough, wheezing, fever, chills or rigors, as well as any orthopnea, leg edema or hemoptysis. Past medical history was significant for tachycardia, orthostatic hypotension, dextrocardia diagnosed 15 years ago, hyperlipidemia and chronic headaches. She had a history of endometrial ablation in May 2007. She had had an uneventful pregnancy 10 years ago. Current medications included nadolol $20 \mathrm{mg}$ twice a day. Family history was not significant for any cardiac or pulmonary disease. She had worked at a childcare center in the past but was now a student. She denied any smoking history and drank 1-2 glasses of wine per week. Review of systems was positive for postnasal drip, nasal congestion and headaches. On examination, vitals were normal. Chest exam showed decreased breath sounds on the right side. There was limited movement of the diaphragm on the right side with respiration. Heart sounds were shifted to the right side. There was no clubbing, cyanosis or edema. A 2D echocardiogram done 15 years ago was interpreted as dextrocardia with normal valves and normal cardiac function. A chest radiograph (fig. 1) done at an outside hospital was read as volume loss on the right side with dextrocardia. Pulmonary function tests including spirometry, lung volumes, and diffusing capacity were within normal limits.

What is your diagnosis?

\section{KARGER}

Fax +41613061234 E-Mail karger@karger.ch www.karger.com
(C) 2009 S. Karger AG, Basel 0025-7931/10/0791-0081\$26.00/0

Accessible online at:

www.karger.com/res

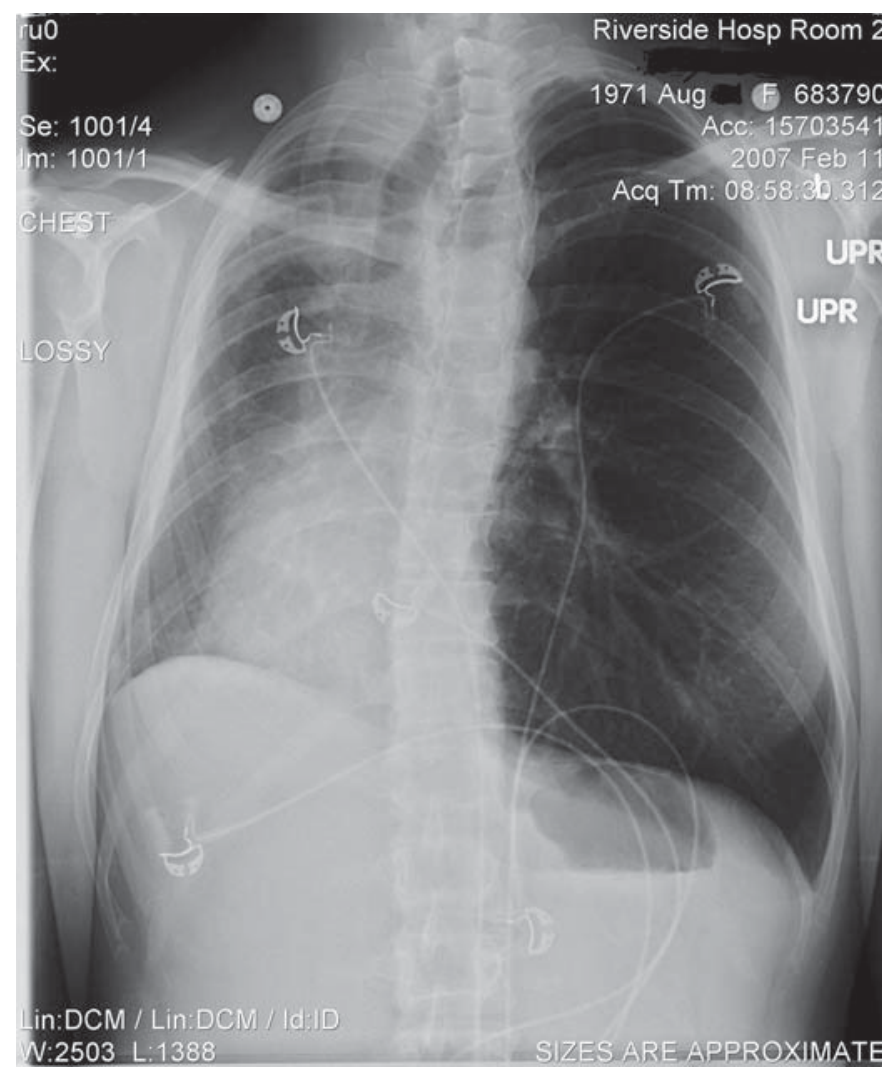

Fig. 1. Chest radiograph showing right lung volume loss with mediastinal shift. 


\section{Diagnosis: Unilateral Absence of a Pulmonary Artery (UAPA) with Right Hypoplastic Lung}

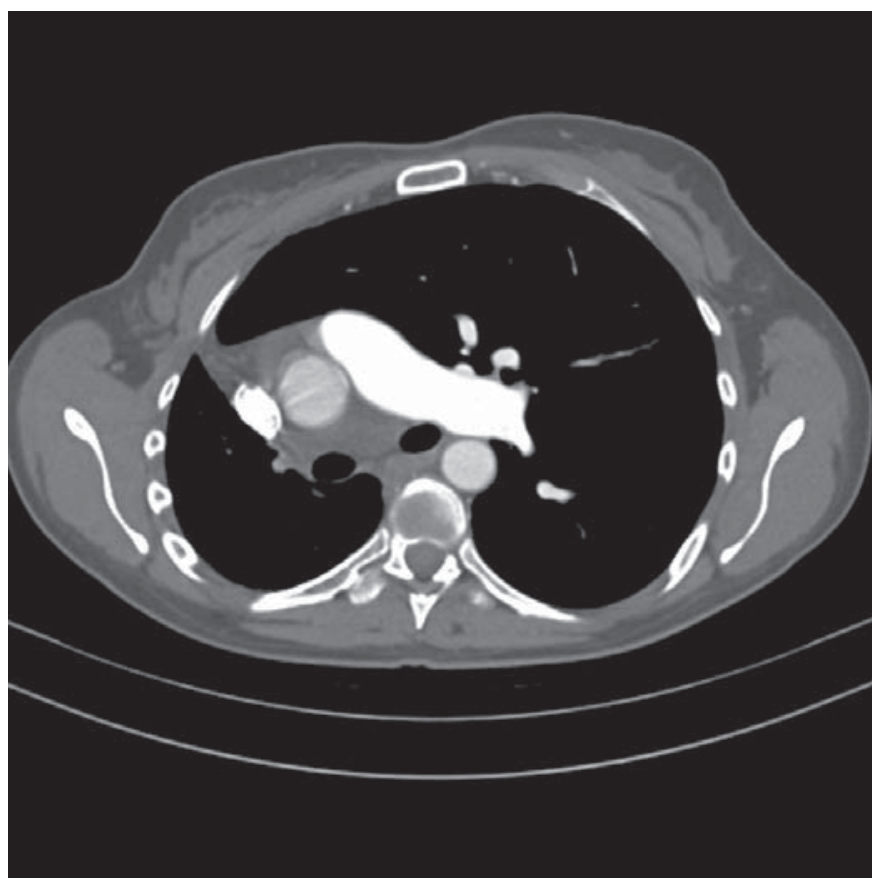

Fig. 2. Contrast-enhanced chest CT of the patient showing the mediastinal vessels.

A contrast-enhanced CT of the chest (fig. 2-5) followed by a repeat $2 \mathrm{D}$ echocardiogram confirmed the absence of a right pulmonary artery along with a right hypoplastic lung. She did not have dextrocardia but had dextroversion due to volume loss in the right chest which mimicked dextrocardia.

\section{Discussion}

UAPA is a rare congenital disorder frequently associated with cardiac abnormalities. This entity was first reported in 1868 [1]. Isolated UAPA (without cardiovascular congenital abnormalities) constitutes about 30-50\% of the cases [2]. Bouros et al. [3] found the prevalence of isolated UAPA to be 1 in 200,000 persons. There is no predilection for either sex. The median age of these patients was 14 years (range $0.1-58$ years) with $12 \%$ of them being infants [2]. Absence of a right pulmonary artery as seen in the present patient is more common than absence of the left pulmonary artery [2]. Most patients are symptomatic and commonly present with recurrent pulmo-

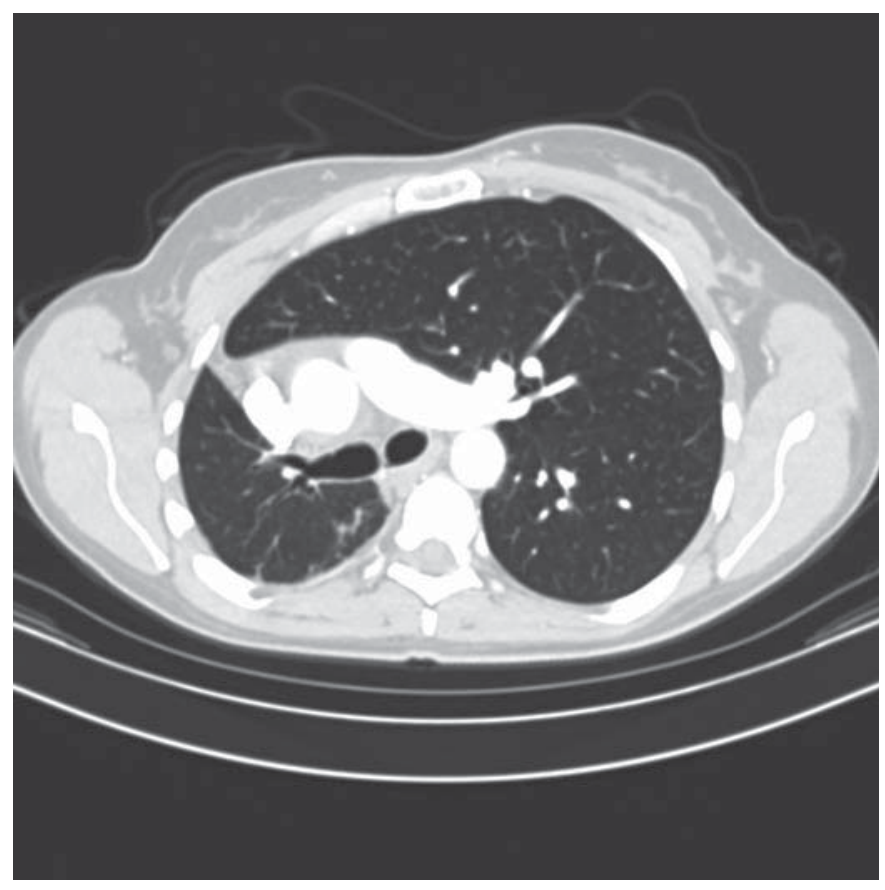

Fig. 3. Chest CT showing hypoplastic right lung.

nary infections, decreased exercise tolerance, hemoptysis or mild exertional dyspnea [4]. About a third of these patients present with recurrent pulmonary infections. Though less common, fatal cases of necrotizing pneumonia can occur and recurrent infections can also lead to bronchiectasis [3, 5, 6]. Bouros et al. [3] suggested that alveolar hypocapnia can cause bronchoconstriction, while impaired mucociliary clearance and the diminished delivery of appropriate inflammatory cells may contribute to the high incidence of infections in patients with UAPA [3]. Hemoptysis is the presenting symptom in $20 \%$ of the patients $[2,7]$. This is due to development of excessive collateral circulation in bronchial, intercostal, subclavian, or subdiaphragmatic arteries [8]. Hemoptysis can be self-limiting for many years, but can lead to massive hemorrhage and death [9]. Pulmonary arterial hypertension (PAH) has been reported in $20-25 \%$ of patients with UAPA $[1,4]$. This is usually unmasked by predisposing factors like high-altitude pulmonary edema (HAPE) and pregnancy [10-14]. The etiology of PAH in these patients is still unclear. 


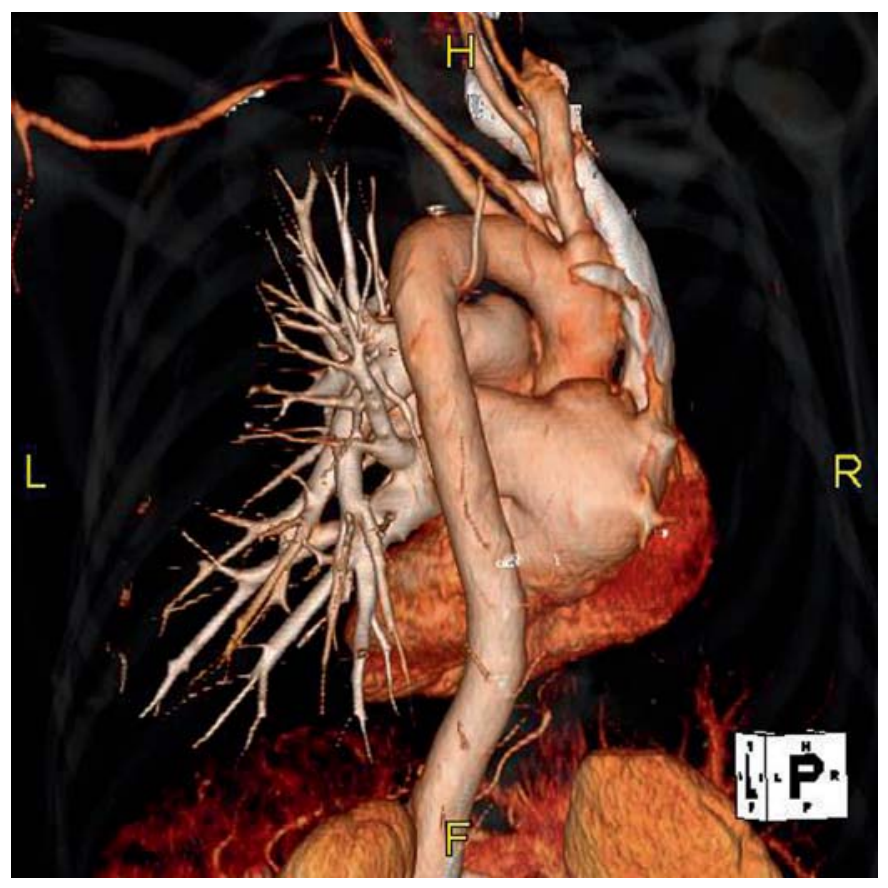

Fig. 4. 3D reconstruction of the chest CT of the patient showing absence of a right pulmonary artery.

Although a chest radiograph usually suggests the diagnosis of UAPA, a contrast-enhanced CT scan or a 2D echocardiogram is needed to confirm the diagnosis. The chest radiograph may show the absence of a hilar shadow, a shrunken affected lung, and a shift of the mediastinal structures to the affected side. In addition, the absence of the left or right pulmonary artery, ipsilateral grossly diminished pulmonary vascular markings, a small hemithorax and intercostal bone space, ipsilateral cardiac and mediastinal displacement, ipsilateral hemidiaphragm elevation, and contralateral lung hyperinflation may also be seen [8]. The echocardiogram also helps exclude other associated cardiac abnormalities and PAH. The most common congenital cardiac malformations associated with UAPA are coarctation of the aorta, either isolated or in combination with a ventricular septal defect; subvalvular aortic stenosis; transposition of the great arteries, either isolated or in combination with ventricular septal defect or pulmonary stenosis; Taussig-Bing malformation and coarctation; congenitally corrected transposition and pulmonary stenosis, and scimitar syndrome [2].

Common causes of mortality (7\%) in isolated UAPA are from massive pulmonary hemorrhage, right heart failure, respiratory failure, PAH or HAPE $[2,9,15]$. PAH

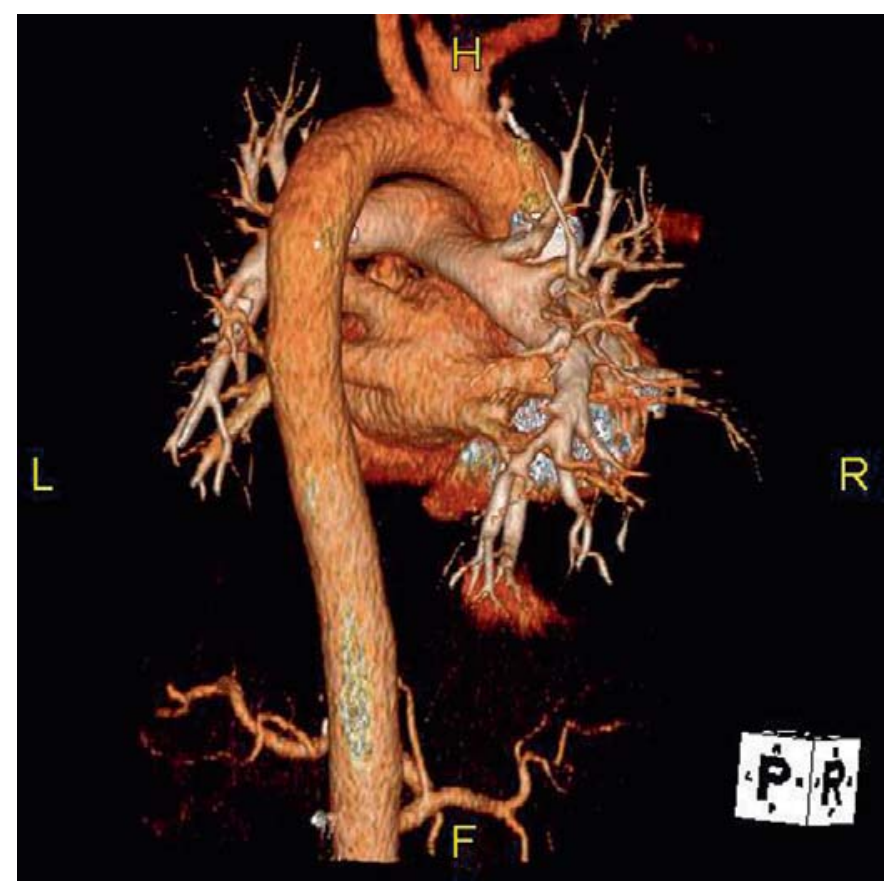

Fig. 5. 3D reconstruction of the chest CT of a normal person compared to that of the patient.

may have devastating effects, especially when it develops during pregnancy. $\mathrm{PAH}$ can be improved by revascularization of the hidden pulmonary artery, although this has only been described in younger patients [16]. When revascularization is proposed, cardiac catheterization is necessary, including pulmonary venous wedge angiography to discover hilar arteries. If revascularization is contraindicated, patients with PAH may benefit from vasodilator treatment used to treat primary PAH. Pneumonectomy or lobectomy is needed for massive hemoptysis, necrotizing bronchopneumonia and bronchiectasis [2].

The present patient had exertional dyspnea without any hemoptysis or evidence of lower respiratory tract infections. Further evaluation included a normal 2D echocardiogram which was followed by a right- and left-heart catheterization. This showed pulmonary artery pressures of $16 / 2$ (mean 8 ) $\mathrm{mm} \mathrm{Hg}$, left-ventricular ejection fraction of $60 \%$ and normal coronaries. A cardiopulmonary exercise test at our facility showed ventilatory limitation. She had a normal heart rate reserve but was limited by dyspnea, which occurred at a reduced maximum $\mathrm{VO}_{2}$ of 1.6 $1 /$ min ( $85 \%$ predicted), maximum work of $120 \mathrm{~W}(78 \%$ predicted) and maximum heart rate of 140 (80\% predicted). However, her ventilation at maximum exercise was $90 \%$ of the calculated maximum voluntary ventilation. 
The likely explanation of this ventilatory limitation is a paradoxical worsening of the dead space ventilation with exercise. This has been reported previously to be a cause of dyspnea in patients with isolated UAPA with normal Dextroversion pulmonary artery pressures [17]. Currently, her symptoms have improved with pulmonary rehabilitation.

\section{References}

-1 Pool PE, Vogel JH, Blount SG Jr: Congenital unilateral absence of a pulmonary artery. The importance of flow in pulmonary hypertension. Am J Cardiol 1962;10:706-732.

-2 Ten Harkel AD, Blom NA, Ottenkamp J: Isolated unilateral absence of a pulmonary artery: a case report and review of the literature. Chest 2002;122:1471-1477.

$\checkmark 3$ Bouros D, Pare P, Panagou P: The varied manifestation of pulmonary artery agenesis in adulthood. Chest 1995;108:670-676.

$\checkmark 4$ Shakibi JG, et al: Isolated unilateral absence of the pulmonary artery. Review of the world literature and guidelines for surgical repair. Jpn Heart J 1978;19:439-451.

$\checkmark 5$ Canver CC, Pigott JD, Mentzer RM Jr: Neonatal pneumonectomy for isolated unilateral pulmonary artery agenesis. Ann Thorac Surg 1991;52:294-295.

6 Werber J, et al: Unilateral absence of a pulmonary artery. Chest 1983;84:729-732.
7 Yiu MW, et al: Hypolucent lung in a woman with recurrent haemoptysis. Respiration 2000;67:341-345.

-8 Lin YM, Liang KW, Ting CT: Unilateral pulmonary artery agenesis with presentation of hemoptysis: a case report. Zhonghua Yi Xue Za Zhi (Taipei) 1999;62:644-647.

$\checkmark$ Thompson JW, et al: Evaluation and management of hemoptysis in infants and children. A report of nine cases. Ann Otol Rhinol Laryngol 1996;105:516-520.

10 Stiller RJ, et al: Agenesis of the pulmonary artery: an unusual cause of dyspnea in pregnancy. Am J Obstet Gynecol 1988;158:172173.

11 Hackett PH, et al: High-altitude pulmonary edema in persons without the right pulmonary artery. N Engl J Med 1980;302:10701073.

12 Ko T, Gatz MG, Reisz GR: Congenital unilateral absence of a pulmonary artery: a report of two adult cases. Am Rev Respir Dis 1990; 141:795-798.
13 Rios B, Driscoll DJ, McNamara DG: Highaltitude pulmonary edema with absent right pulmonary artery. Pediatrics 1985;75:314317.

14 Sebbane M, et al: Unilateral agenesis of the pulmonary artery and high-altitude pulmonary edema (HAPE) at moderate altitude. Pediatr Pulmonol 1997;24:111-114.

15 Ferrari M, et al: Effect of changing position on arterial oxygenation in a patient with agenesia of the left pulmonary artery. Respiration 1997;64:371-374.

16 Toews WH, Pappas G: Surgical management of absent right pulmonary artery with associated pulmonary hypertension. Chest 1983; 84:497-499.

17 Brassard JM, Johnson JE: Unilateral absence of a pulmonary artery. Data from cardiopulmonary exercise testing. Chest 1993;103: 293-295. 